\title{
Nanodiscs as a therapeutic delivery agent: inhibition of respiratory syncytial virus infection in the lung
}

This article was published in the following Dove Press journal:

International Journal of Nanomedicine

12 April 2013

Number of times this article has been viewed

\author{
Mari Numata' \\ Yelena V Grinkova ${ }^{2}$ \\ James R Mitchell' \\ Hong Wei Chu' \\ Stephen G Sligar ${ }^{2}$ \\ Dennis R Voelker \\ 'Department of Medicine, Program \\ in Cell Biology, National Jewish \\ Health, Denver, CO, USA \\ ${ }^{2}$ Department of Biochemistry, \\ University of Illinois, Urbana, \\ IL, USA
}

\begin{abstract}
There is increasing interest in the application of nanotechnology to solve the difficult problem of therapeutic administration of pharmaceuticals. Nanodiscs, composed of a stable discoidal lipid bilayer encircled by an amphipathic membrane scaffold protein that is an engineered variant of the human Apo A-I constituent of high-density lipoproteins, have been a successful platform for providing a controlled lipid composition in particles that are especially useful for investigating membrane protein structure and function. In this communication, we demonstrate that nanodiscs are effective in suppressing respiratory syncytial viral (RSV) infection both in vitro and in vivo when self-assembled with the minor pulmonary surfactant phospholipid palmitoyloleoylphosphatidylglycerol (POPG). Preparations of nanodiscs containing POPG (nPOPG) antagonized interleukin-8 production from Beas2B epithelial cells challenged by RSV infection, with an $\mathrm{IC}_{50}$ of $19.3 \mu \mathrm{g} / \mathrm{mL}$. In quantitative in vitro plaque assays, $\mathrm{nPOPG}$ reduced RSV infection by $93 \%$. In vivo, nPOPG suppressed inflammatory cell infiltration into the lung, as well as IFN- $\gamma$ production in response to RSV challenge. $\mathrm{nPOPG}$ also completely suppressed the histopathological changes in lung tissue elicited by RSV and reduced the amount of virus recovered from lung tissue by $96 \%$. The turnover rate of nPOPG was estimated to have a halftime of 60-120 minutes (m), based upon quantification of the recovery of the human Apo A-I constituent. From these data, we conclude that $\mathrm{nPOPG}$ is a potent antagonist of RSV infection and its inflammatory sequelae both in vitro and in vivo.
\end{abstract}

Keywords: nanodiscs, therapeutic delivery, anti-viral, innate immunity, phospholipids

\section{Introduction}

Nanodiscs are self-assembled discoidal particles that are a phospholipid bilayer stabilized by an encircling helical protein "belt" called a membrane scaffold protein (MSP) (Figure 1). ${ }^{1-3}$ The MSP renders the entity soluble and biocompatible due to its amphipathic nature: non-polar residues face inward to mate with the acyl chains of the phospholipids, and polar/charged residues are at the aqueous interface. The MSP sequence was derived from the major protein component of the human high-density lipoprotein Apo A-I. ${ }^{4-11}$ While the primary circulating form of the high-density lipoprotein is a spherical particle with cholesterol esters in the core, its transitory, nascent form is discoidal. ${ }^{12-14}$ A nanometer-scale membrane mimetic would have great utility in the investigation of biological processes involving membrane surfaces and associated membrane proteins. The necessity of obtaining homogeneous and monodisperse particles in order to apply the broad spectrum of biochemical and biophysical techniques to the study of integral membrane proteins assembled into nanodiscs required extensive protein engineering of the encircling Apo A-I protein. This effort resulted in a series
Correspondence: Stephen G Sligar Department of Biochemistry, School of Molecular and Cellular Biology, University of Illinois, 505 S Goodwin Avenue, Urbana, IL 6180I, USA

Tel +I 2172447395

Email s-sligar@illinois.edu 


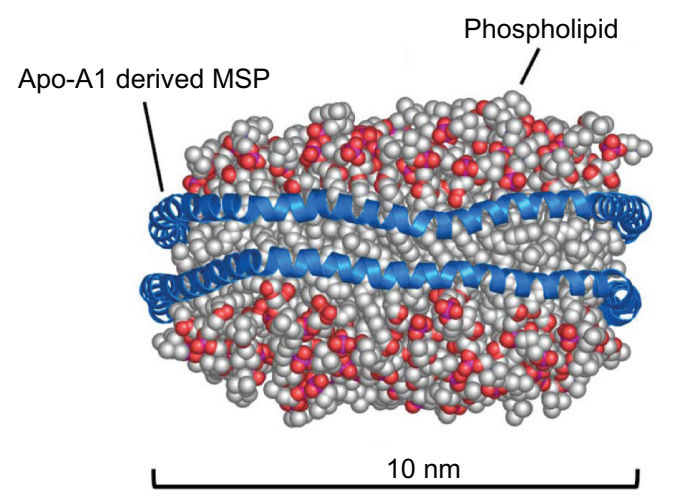

Figure I Molecular dynamic simulated model of Nanodisc. ${ }^{30}$

Notes: Nanodiscs consist of a phospholipid bilayer encircled by two molecules of an Apo-Al-derived membrane scaffold protein, which organizes the lipids and shields the acyl chains from the aqueous environment.

Abbreviation: MSP, membrane scaffold protein.

of amphipathic helical MSPs that could be used to generate discoidal bilayer particles of varying size, currently $\sim 9 \mathrm{~nm}$ to $\sim 17 \mathrm{~nm}$, where the length of the MSP determines nanodisc size. ${ }^{1-3}$ The resultant family of nanodiscs has seen broad application for the structural and functional studies of membrane proteins, following the discovery that detergentsolubilized membrane proteins could be self-assembled into the nanodisc by simple detergent removal with the target in a native-like lipid environment with "native structural presentation," yet soluble in aqueous solutions. ${ }^{15,16}$ Numerous laboratories have taken advantage of nanodiscs, as has been summarized in recent reviews. ${ }^{17-19}$ The human Apo A-I origin of MSPs suggests that nanodiscs could be useful for in vivo use as therapeutic delivery agents. In this communication, we demonstrate that nanodiscs can deliver therapeutic lipids to the lung, prevent viral infection, and suppress the ensuing deleterious inflammatory response.

Respiratory syncytial virus (RSV) is one of the most common pathogens affecting young children, with infection rates over $90 \%$ between birth and age two. ${ }^{20}$ There is also growing appreciation that RSV causes serious respiratory illness in the elderly and high-risk adults with chronic pulmonary diseases such as asthma and chronic obstructive pulmonary disease. ${ }^{21,22}$ There is currently no vaccine or clinically approved treatment for RSV infection. ${ }^{20-22}$ Recent studies demonstrate that the minor pulmonary surfactant phospholipid, palmitoyloleoylphosphatidylglycerol (POPG), markedly attenuates RSV infection in vivo and in vitro. ${ }^{23}$ The primary action mechanism of POPG appears to be the inhibition of virus binding to epithelial cells. ${ }^{23}$ Unfortunately, there are limited means to deliver potentially therapeutic phospholipids in vivo, particularly to the alveolar compartment in the lung. Liposomes offer one potential vehicle, although many problems with formulation exist, including overall in vivo stability, preparation homogeneity, and the drug composition shelf-life. Nanodiscs can be generated with precise control over lipid content and particle size, can be lyophilized and reconstituted, and can be made pyrogen-free and antigenically neutral. In this study, we investigated the use of POPG nanodiscs as a means of introducing therapeutic lipids for the prevention of viral infection by RSV. We examined the potency and effectiveness of nPOPG as an antagonist of RSV infection using both in vitro and in vivo systems. We quantified the potency of nPOPG against RSV infection in vitro by measuring interleukin-8 (IL-8) production and viral plaque formation. In addition, we determined the effectiveness of nPOPG using a mouse model of RSV infection and estimated the stability of nPOPG in the lung. Our findings show the utility of nanodiscs as a therapeutic delivery platform and demonstrate the effective inhibition of RSV infection both in vitro and in vivo.

\section{Methods}

\section{Virus preparation, cell culture, and RSV infection in vitro}

The human RSV A2 strain was obtained from the American Tissue Culture Collection (VR-1540, Manassas, Virginia, USA). The virus was propagated in HEp2 cells (ATCC) with culturing in DMEM/F12 (Invitrogen, Camarillo, CA, USA), supplemented with 5\% bovine growth serum (BGS; Hyclone, South Logan, UT, USA). RSV was purified using methods previously described, under endotoxin-free conditions. ${ }^{25,26}$ Viral titers and growth were measured by quantitative plaque assays, as previously described..$^{23,27}$

Beas2B cells were obtained from ATCC and cultured using a BEBM-bronchial epithelial cell growth medium (Lonza, Walkersville, MD, USA). Cells were infected at a multiplicity of infection (MOI) of 0.5 and incubated for 48 hours. Phospholipids were obtained from Avanti (Alabaster, AL, USA). Prior to RSV infection, cells were pre-incubated with nPOPG and nPOPC (200 $\mu \mathrm{g}$ phospholipid/mL) for $1 \mathrm{~h}$, with RSV subsequently added to the cultures. Beas2B cells were also stimulated with $10 \mathrm{ng} / \mathrm{mL}$ of the TLR5 agonist flagellin (Alexis Biochemicals USA, San Diego, California, USA), with or without liposome POPG, nPOPG, or nPOPC (200 $\mu \mathrm{g}$ phospholipid $/ \mathrm{mL}$ ) for $48 \mathrm{~h}$. IL-8 release into the culture supernatants was determined by commercial ELISA assay (Invitrogen). The $\mathrm{IC}_{50}$ values for $\mathrm{nPOPG}$ were determined from the inspection of best-fit curves for the data points. 
The cytopathic effects of the cultures were examined by light microscopy.

\section{Nanodisc preparation}

The MSP used for self-assembling phospholipids into nanodiscs was expressed and purified as described previously. ${ }^{1,2}$ For this work we used MSP1D1 with a heptahistidine N-terminal affinity tag, which forms nanodiscs with a diameter of $9.7 \mathrm{~nm}$, containing approximately 125 POPC or POPG lipids per disc. Additional purification steps were used to reduce the endotoxin level of the protein preparation: the MSP preparation (100-150 mg) was adsorbed onto a $1 \mathrm{~cm} \times 5 \mathrm{~cm}$ Ni-NTA column and washed with 10 bed volumes of an aseptically prepared $1 \%$ Triton X-100 solution in phosphate buffered saline (PBS), followed by 10 bed volumes of buffer without detergent. The MSP was eluted with a 0.3-M imidazole solution and the buffer exchanged using an Amicon 15 centrifugal ultrafiltration concentrator. The sample was filter-sterilized and the endotoxin level determined using a PYROGENT-5000 assay (Lonza, Walkersville, MD, USA).

Nanodiscs composed of phospholipids and the scaffold protein MSP1D1 were prepared as previously described. .,2,17 $^{2}$ Briefly, cholate-solubilized phospholipids and purified MSP1D1 were combined and sonicated to form clear solutions at molar ratios of 130:65:1 (cholate:POPC or POPG:MSP1D1). The cholate was removed from the preparations with Amberlite XAD-2. The sample integrity was confirmed by size-exclusion chromatography using a Superdex 200 10/300 GL column. In all samples, more than 95\% of material was eluted as a single peak, with the retention time corresponding to a Stokes diameter of $9.7 \pm 0.2 \mathrm{~nm}$, which is consistent with previously published results. ${ }^{2}$

\section{Measurement of mRNA-encoding RSV F-protein by qRT-PCR}

HEp2 cells were cultured in 24-well plates and inoculated for 2 hours at $37^{\circ} \mathrm{C}$ with RSV at an MOI of $1 \times 10^{-3}$, in either the absence or presence of $200 \mu \mathrm{g} / \mathrm{mL}$ nPOPG or nPOPC. Following the adsorption period, the cells were washed three times with PBS and then shifted to a culture medium either with or without the nanodiscs. Immediately following adsorption, and at 24-96 hours, total well contents were processed for RNA extraction using a Qiagen RN-easy kit (Qiagen, Germantown, Maryland, USA). The mRNA-encoding F-protein (F mRNA) was quantified using a qRT-PCR kit (Invitrogen). PCR products were also qualitatively analyzed by gel electrophoresis. The primers for $\mathrm{F}$ protein mRNA were $5^{\prime}$ GGAGGAAGTCTTAGCATATGTAG 3' and 5' CCATCCTCTGTCGGTTC 3'.
We calculated gene expression levels using the manufacturer's software (Bio-Rad, Hercules, CA, USA).

\section{Binding of RSV to epithelial cells}

For cellular binding studies, HEp2 cells were grown in 24-well plates and RSV was adsorbed to the monolayers at varying MOIs $(0,1,2,5$, and 10$)$ for 2 hours at $18^{\circ} \mathrm{C}$, either in the absence or presence of nPOPG, nPOPC, or liposome POPG (200 $\mu \mathrm{g} / \mathrm{mL}$ of phospholipids). At $18^{\circ} \mathrm{C}$, endocytosis by HEp 2 cells is minimal, and this temperature allows viral binding to reach equilibrium within 2 hours. ${ }^{28}$ The cell monolayers were processed at $0^{\circ} \mathrm{C}$ for subsequent analysis by quantitative immunoblotting using a goat anti-RSV antibody (AbD Serotec, Raleigh, NC, USA).

\section{In vivo measurements of $n P O P G$ effects on RSV infection}

Six-week-old female BALB/c mice were obtained from Jackson Laboratory (Bar Harbor, ME, USA). Mice were anesthetized and inoculated in a final volume adjusted to $50 \mu \mathrm{L}$. The RSV infection contained $1 \times 10^{7} \mathrm{pfu} / 50 \mu \mathrm{L}$ in PBS. nPOPG was adjusted to $400 \mu \mathrm{g}$ phospholipid in a total volume of $50 \mu \mathrm{L}$ of PBS. For lipid antagonism, RSV and nPOPG were mixed together in PBS prior to inoculation. As described previously, all in vivo RSV effects required a replication-competent virus and were eliminated upon ultraviolet light inactivation of the virus. ${ }^{23}$ At 5 days after infection, mice were euthanized by intraperitoneal injection of $0.25 \mathrm{~mL}$ of Nembutal $(10 \mathrm{mg} / \mathrm{mL})$. All animal experiments followed prescribed guidelines and were approved by the Institutional Animal Care and Use Committee.

Euthanized mice were first dissected to expose the trachea and intubated with a cannula. The lungs were then lavaged with $1 \mathrm{~mL}$ of PBS. Next, the thorax was opened and the lungs were removed. The left lungs were homogenized in a DMEM tissue culture medium (Lonza, Walkersville, MD), and extracts were centrifuged at $2000 \mathrm{~g}$ for 15 minutes at $0^{\circ} \mathrm{C}$ and used for quantitative plaque assay analysis. Lower right lungs were fixed overnight in $10 \%$ buffered formalin, subsequently dehydrated, and the tissues paraffin-embedded. Four- $\mu$ m-thick sections were prepared and stained with Hematoxylin-Eosin. Tissues were evaluated using a histopathologic inflammatory scoring system, as described previously. ${ }^{24}$ The lavage fluid was used for total cell counting, and differential cell counts were performed on cytospin preparations stained with trypan blue, as previously described. ${ }^{23}$ The cell-free supernatants resulting from the centrifugation of bronchoalveolar lavage fluid (BALF) 
were used for the measurement of IFN- $\gamma$ by ELISA (BD Bioscience, San Diego, CA, USA).

\section{Measurements of nPOPG turnover}

Anesthetized mice were inoculated with $50 \mu \mathrm{L}$ nanodisc preparations containing $400 \mu \mathrm{g}$ of POPG. Following inoculation, the mice were euthanized at 0 minutes, 30 minutes, 60 minutes, 120 minutes, and 360 minutes. The euthanized mice were subjected to lavage with the thorax closed. Each animal received a $1-\mathrm{mL}$ lavage of PBS. The recovered lavage material was subjected to SDS-PAGE and quantitative western blotting with an anti-Apo-A1 antibody (Calbiochem, La Jolla, CA, USA).

\section{Statistical analysis}

All results are shown as means \pm SE. ANOVA was used to determine the level of difference between all groups. Differences were considered to be significant at $P<0.05$.

\section{Results}

\section{nPOPG attenuates RSV-induced IL-8 production in human epithelial cells}

IL-8 is produced by many types of epithelial cells in response to different infectious challenges and serves as an excellent biochemical marker for inflammatory processes. We compared the effects of nPOPG on RSV-induced IL-8 production in a human bronchial epithelial cell line (Beas2B). After 1 hour pre-incubation with either liposome POPG or nPOPG (both at $200 \mu \mathrm{g} / \mathrm{mL}$ phospholipid), Beas $2 \mathrm{~B}$ cells were infected with RSV at an MOI of 0.5 virus particles/cell for a period of $48 \mathrm{~h}$. RSV infection induced a 100 -fold increase in IL-8 production; liposome POPG inhibited this response by $95.4 \%$, and $\mathrm{nPOPG}$ treatment suppressed this induction of IL-8 by $97.4 \%$ (Figure 2A). Liposomes and nanodiscs assembled with a control lipid, palmitoyloleoylphosphatidyl choline (POPC), which contains the same hydrophobic diacylglycerol moiety as POPG but differs in the hydrophilic head group, did not significantly inhibit IL-8 production. When Beas2B cells were challenged with the Toll-like receptor 5 ligand flagellin, they also produced a robust IL-8 response, but nPOPG, nPOPC, and liposome POPG were all ineffective at suppressing this inflammatory response (Figure 2B). These latter results demonstrate that the actions of nPOPG and liposome POPG are not non-specific, or cytotoxic, and do not generally suppress all inflammatory signaling pathways for the production of IL-8.

For a more detailed comparison of the potency of nPOPG, we measured IL-8 production induced by the virus with varying doses of the lipid. The results from these experiments reveal that nPOPG exerts its effect in a concentration-dependent manner, with an $\mathrm{IC}_{50}$ of $19.1 \pm 6.1 \mu \mathrm{g} / \mathrm{mL}$ and maximal inhibition at approximately $100 \mu \mathrm{g} / \mathrm{mL}$ (Figure 3A). Liposome POPG produces a similar $\mathrm{IC}_{50}$ at $12.3 \pm 3.8 \mu \mathrm{g} / \mathrm{mL}$. In contrast to $\mathrm{nPOPG}$ and liposome POPG, treatment with $\mathrm{nPOPC}$ at $200 \mu \mathrm{g} / \mathrm{mL}$ had a minimal inhibitory effect upon IL-8 production. Microscopic examination of the cytopathic effects of RSV is shown in Figure 3B. Control cells show a typical monolayer of Beas $2 \mathrm{~B}$ cells. Treatment with RSV added at an MOI of 0.5 for $48 \mathrm{~h}$ produces a cell layer with significantly reduced cell numbers, as well as cells with a hypertrophic,
A

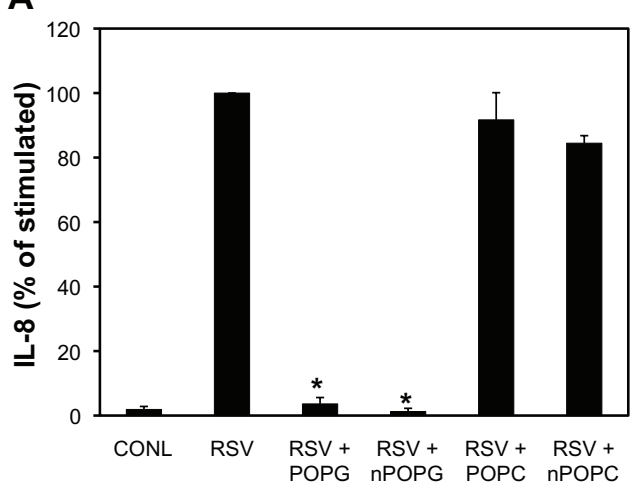

B

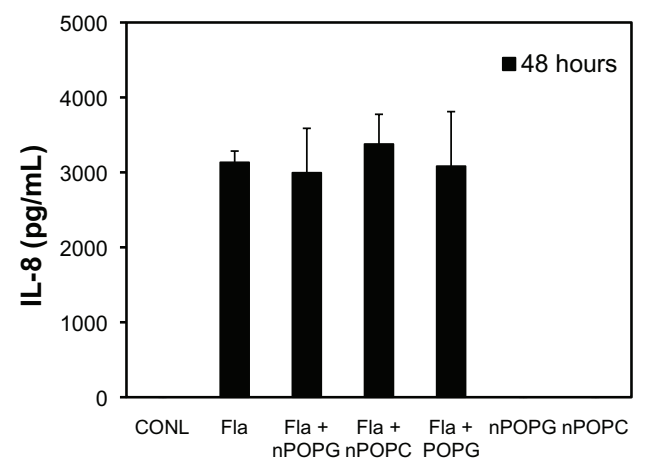

Figure 2 nPOPG inhibits RSV-induced IL-8 production by Beas2B cells. (A) IL-8 production by the Beas2B cells was measured by ELISA after either sham treatment or virus infection for 48 hours, in either the absence or presence of $200 \mu \mathrm{g} / \mathrm{mL}$ POPG, POPC added in the form of liposomes (+POPG), or nanodiscs (+nPOPG, +nPOPC). The cells were infected at a multiplicity of infection (0.5). Values shown are means \pm SE for three independent experiments. *indicates $P<0.00 \mathrm{I}$. The average production of IL-8 with RSV infection alone in the three experiments was $3977.6 \pm 865 \mathrm{pg} / \mathrm{mL}$. (B) Flagellin $(10 \mathrm{ng} / \mathrm{mL})$-induced IL-8 production by the Beas $2 B$ cells was measured by ELISA after 48 hours of incubation. The flagellin challenge was performed in either the absence or presence of $200 \mu g / \mathrm{mL}$ of nPOPG, nPOPC, or liposomes (+POPG), as indicated. The treatment of the cells with nPOPG or nPOPC in the absence of flagellin challenge is also shown in the figure.

Note: The data shown are from three independent experiments.

Abbreviations: CONL, uninfected control; ELISA, enzyme linked immunosorbent assay; Fla, flagellin; POPG, liposomal palmitoyl-oleoyl-phosphatidylglycerol; nPOPG, nanodisc palmitoyl-oleoyl-phosphatidylglycerol; POPC, liposomal palmitoyl-oleoyl-phosphatidylcholine; nPOPC, nanodisc palmitoyl-oleoyl-phosphatidylcholine; RSV, respiratory syncytial virus; SE, standard errors of the mean. 
A

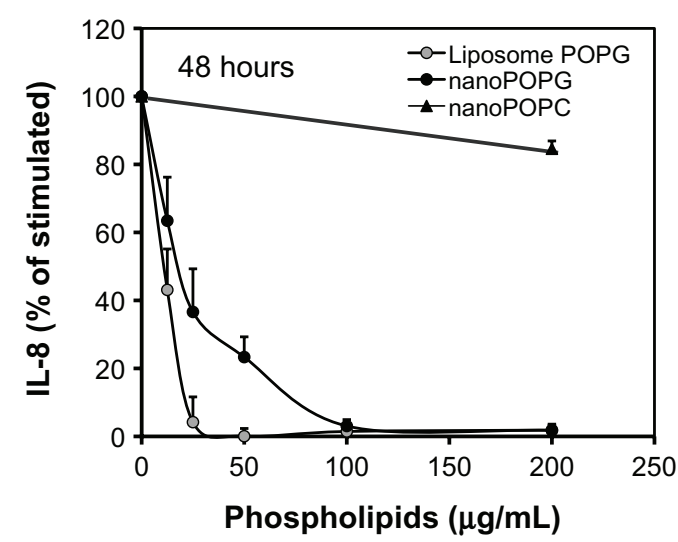

B
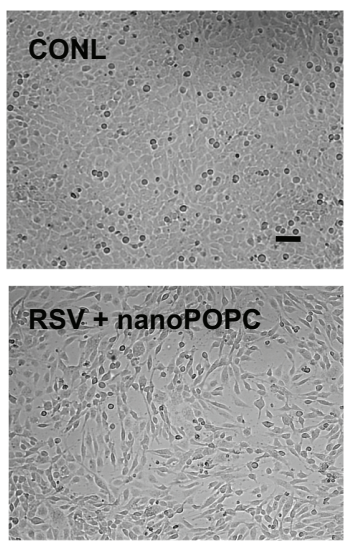

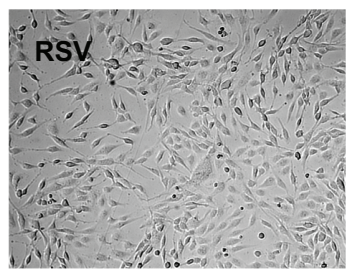

RSV + nanoPOPG

Figure 3 Nanodisc POPG suppresses RSV-elicited IL-8 production in a concentration- and phospholipid class-dependent manner and inhibits the cytopathic effects of RSV upon Beas2B cells. (A) IL-8 production by Beas2B cells was measured by ELISA after challenging cultures with RSV (multiplicity of infection $=0.5$ ) for 48 hours in either the absence or presence of varying concentrations of liposome POPG, nPOPG, or nPOPC. At the end of the incubation period, the culture medium was harvested and analyzed for the presence of secreted IL-8. Data shown are the means \pm SE for three experiments. (B) Beas2B cells were either sham-treated or infected with a virus (RSV) for 48 hours, in either the absence or presence of $200 \mu \mathrm{g} / \mathrm{mL}$ nPOPG or nPOPC, as indicated. The cell monolayers were visualized by phase contrast microscopy.

Note: The bar inset in the CONL panel corresponds to $50 \mu \mathrm{m}$.

Abbreviations: CONL, uninfected control; POPG, palmitoyl-oleoyl-phosphatidylglycerol; nanoPOPG, nanodisc palmitoyl-oleoyl-phosphatidylglycerol; nanoPOPC, nanodisc palmitoyl-oleoyl-phosphatidylcholinel; RSV, respiratory syncytial virus; SE, standard errors of the mean.

spindle-shaped morphology. Treatment of cells with RSV and nPOPC produces a cell layer that is quite similar in appearance to the virus-only treatment. In contrast, treatment of cells with RSV and nPOPG produces a cell monolayer that is nearly indistinguishable from control cells. Previously published micrographs also show that liposome POPG abrogates the cytopathic effects of RSV. ${ }^{23}$ These data provide morphological evidence that POPG protects the epithelial cells from the cytopathic effects of RSV infection. From the above findings, we conclude that $\mathrm{nPOPG}$ is an effective antagonist of the RSV-induced inflammatory and cytopathic response of Beas2B cells.

\section{nPOPG treatment inhibits RSV infection and in vitro plaque formation}

We examined the ability of nPOPG to disrupt the RSV infection cycle by quantifying viral plaque formation with HEp2 cells in either the absence or presence of liposomes or nanodiscs reconstituted with POPG. Liposomes or nanodiscs were added 1 hour prior to the viral challenge, at a phospholipid concentration of $200 \mu \mathrm{g} / \mathrm{mL}$. RSV, at titers of $2 \times 10^{4}-2 \times 10^{-2} / \mathrm{mL}$, was allowed to adsorb to the cell monolayers for 1 hour with subsequent removal of the lipid and virus. These cultures were overlaid with agar and the formation of plaques was measured after 5 days. The liposome POPG inhibited plaque formation by $>99 \%$ and the nPOPG attenuated plaque formation by $93 \%$ (Figure 4 ). These results show that $\mathrm{nPOPG}$ is effective as an in vitro inhibitor of RSV infection.

\section{nPOPG interferes with viral propagation from the earliest stages of infection}

Monolayers of HEp2 cells were infected at a low MOI, and the infection was followed for 4 days by using qRT-PCR to measure the mRNA encoding the viral F-protein. As shown in Figure 5, viral mRNA was detected after 24 hours of infection and continued to increase for another 72 hours. The inclusion

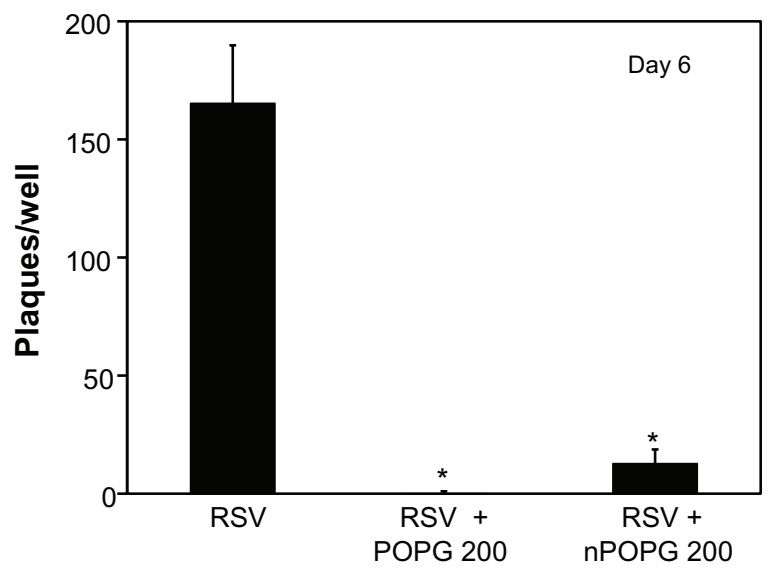

Figure 4 nPOPG inhibits RSV infection of HEp2 cells in tissue culture.

Notes: Monolayers of HEp2 cells were incubated in six well plates for I h prior to the addition of RSV either without lipids or with $200 \mu \mathrm{g}$ of liposome POPG or nPOPG. Serial 10 -fold dilutions of RSV from $2 \times 10^{4}-2 \times 10^{2}$ infectious particles per $\mathrm{mL}$ were added to duplicate wells in the absence or presence of lipids. After a viral adsorption period of $\mathrm{I} h$, the unbound virus and the lipid were removed from each well, and the monolayers were overlaid with an agar-containing medium for the development of plaques. After $6 \mathrm{~d}$, the cultures were fixed with $1 \%$ buffered formalin and stained with $0.05 \%$ neutral red, and plaques were counted. The data shown are means \pm SE from three independent experiments. *indicates $P<0.001$. Abbreviations: POPG, liposomal palmitoyl-oleoyl-phosphatidylglycerol; nPOPG, nanodisc palmitoyl-oleoyl-phosphatidylglycerol; RSV, respiratory syncytial virus; SE, standard errors of the mean. 

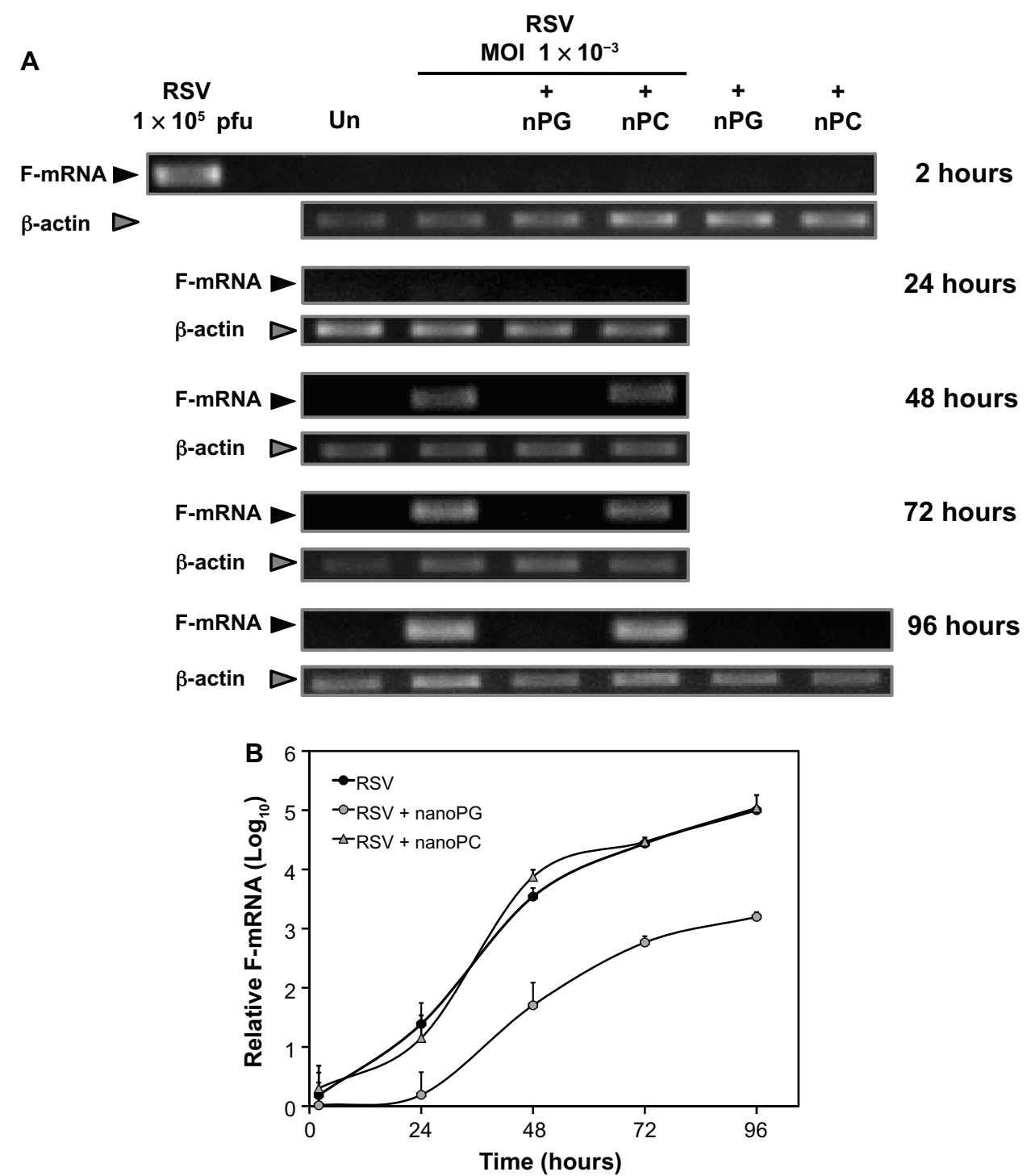

Figure 5 nPOPG inhibits the production of RSV mRNA. (A) Monolayers of HEp2 cells were pretreated with $200 \mu \mathrm{g} / \mathrm{mL} \mathrm{nPOPG}$ or nPOPC for I h prior to the addition of RSV (multiplicity of infection $=1 \times 10^{-3}$ ). After $2 \mathrm{~h}$ of RSV adsorption in the absence or presence of lipids, the cells were washed three times with PBS and then grown in DMEM/FI2, either with or without $200 \mu \mathrm{g} / \mathrm{mL} \mathrm{nPOPG}$ or nPOPC. The complete contents of each well were processed for RNA extraction at various time points (from $2 \mathrm{~h}$ to $96 \mathrm{~h}$ ) and subjected to analysis by quantitative RT-PCR and gel electrophoresis. (B) The results of two independent experiments are shown.

Note: Values are means \pm SD.

Abbreviations: MOI, multiplicity of infection; nPG, nanodisc palmitoyl-oleoyl-phosphatidylglycerol; nPC, nanodisc palmitoyl-oleoyl-phosphatidylcholine; F-mRNA, messenger RNA encoding viral F-glycoprotein; RSV, respiratory syncytial virus.

of nPOPC with the RSV inoculum did not alter the growth of the virus. In contrast, the addition of nPOPG with the virus at the time of infection markedly suppressed the appearance and amount of viral RNA. At the end of the 96-hours growth period, the nPOPG-treated cultures expressed less than $1 \%$ of the RSV mRNA found in cells infected with the virus alone, or the virus plus nPOPC. These data demonstrate that nPOPG treatment markedly suppresses RSV gene expression and replication.

\section{nPOPG blocks RSV}

\section{attachment to epithelial cells}

To determine the site of nPOPG action as an antagonist of viral infection, we performed RSV binding experiments with monolayers of HEp2 cells in either the absence or presence of nPOPG, nPOPC, or liposome POPG. The attachment of the virus to the cell surface was quantified by recovering washed cell monolayers and performing electrophoresis and immunoblotting to detect viral proteins. The binding experiments were performed at $18^{\circ} \mathrm{C}$ to minimize endocytosis. The data in Figure 6A, show the immunoblot analysis of either uninfected cells or cells incubated with varying concentrations of virus, with or without nanodiscs or liposomes. The data in Figure 6B summarize the findings from three independent experiments. Virus attachment to the cell surface is a high-affinity, saturable process. The addition of nPOPC to the binding reaction is without significant effect. In contrast, 
A

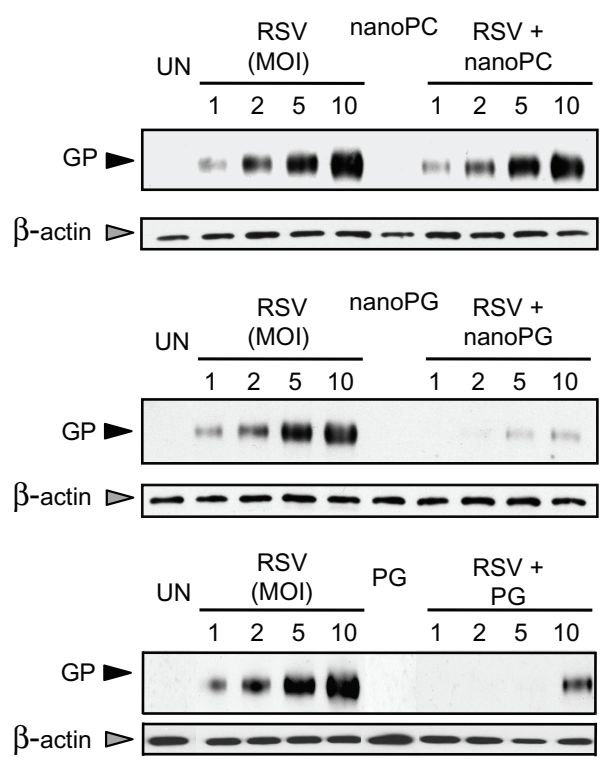

B

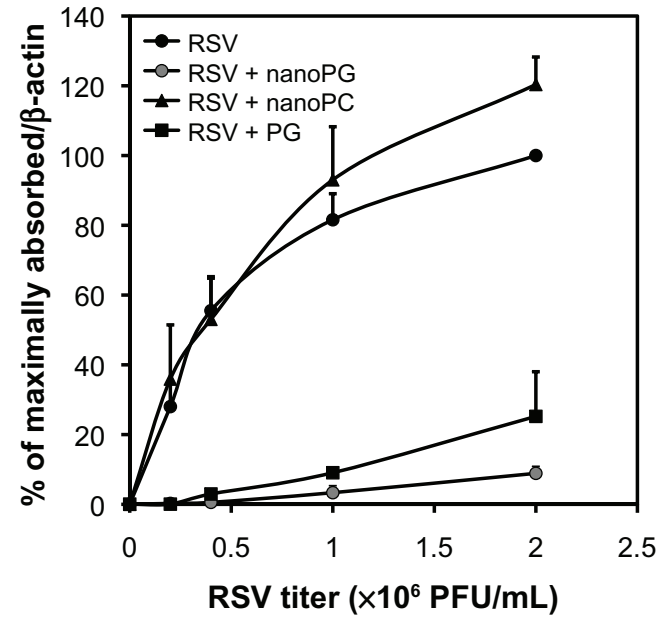

Figure 6 nPOPG blocks RSV binding to epithelial cells. (A) Monolayers of HEp2 cells, at $18^{\circ} \mathrm{C}$, were challenged with RSV at $\mathrm{MOI}=0-10$, as indicated, in either the absence or presence of $200 \mu \mathrm{g} / \mathrm{mL}$ nPOPC, nPOPG, or liposome POPG. UN identifies cultures that were not infected with the virus. The cultures were washed with PBS three times and harvested and processed for SDS-PAGE and immunoblotting. GP indicates the location of the viral G protein, and $\beta$-actin blotting was used to normalize the recovery of cells from each well. (B) The quantification of three independent immunoblotting experiments is shown.

Note: Values are means \pm SE for three independent experiments.

Abbreviations: MOI, multiplicity of infection; nanoPG, nanodisc palmitoyl-oleoyl-phosphatidylglycerol; nanoPC, nanodisc palmitoyl-oleoyl-phosphatidylcholine; UN, unifected control; GP, viral G-glycoprotein; PFU, plaque forming units; RSV, respiratory syncytial virus; SE, standard errors of the mean.

the inclusion of nPOPG suppresses RSV attachment to epithelial cells by $93 \%$ (at an MOI of 10), and liposome POPG inhibits this binding by $75 \%$ (at an MOI of 10 ).

\section{Intranasal treatment of mice with nPOPG attenuates RSV infection in vivo}

We utilized an in vivo infection model to examine RSV infection following intranasal administration of nPOPG. Mice were intranasally $\left(1 \times 10^{7} \mathrm{pfu} /\right.$ mouse $)$ inoculated with RSV, either in the absence or presence of liposome POPG or nPOPG containing $400 \mu \mathrm{g} / \mathrm{mL}$ phospholipid. Mice were euthanized 5 days after infection and their lungs were lavaged with $1 \mathrm{~mL}$ of saline. The recovered BALF was used for the analysis of total cell numbers, inflammatory cell populations, and IFN- $\gamma$ production. The left lungs were harvested and processed for quantitative plaque assays to determine viral titers. The right lungs were processed for lung histology, and the degree of pathology was determined as previously described. ${ }^{23,24}$

As shown in Figure 7A, RSV infection produced a 2.6fold increase in the number of cells obtained by bronchoalveolar lavage, when compared with sham-treated mice $\left(\mathrm{CONL}=0.52 \pm 0.1 \times 10^{5} \mathrm{cell} / \mathrm{mL}, \mathrm{RSV}=1.31 \pm 0.19 \times 10^{5}\right.$ cell $/ \mathrm{mL}$ ). Liposome POPG and nPOPG treatment markedly reduced the total cells recovered in BALF after RSV infection (liposomes $=0.45 \pm 0.04 \times 10^{5}$ cells $/ \mathrm{mL}$ and nanodiscs $=0.56 \pm 0.06 \times 10^{5}$ cells $/ \mathrm{mL}$ ). Inflammatory cell infiltrates induced by RSV consist mainly of neutrophils and lymphocytes, which are recovered in BALF. These inflammatory cell populations were significantly attenuated by treatment with liposome POPG and nPOPG, in conjunction with the RSV challenge (Figure 7B). IFN- $\gamma$ production occurs as part of the host anti-viral response induced by RSV but is undetectable in uninfected animals. The robust IFN- $\gamma$ response induced by RSV was completely eliminated in mice receiving either liposome POPG or nPOPG along with the virus (Figure 7C). The loss of the IFN- $\gamma$ response occurring with $n P O P G$ treatment is a result of lipid inhibition of the viral infection process. The production of infectious virus particles after the initial infection was quantified by performing plaque assays on lung homogenates. nPOPG suppressed the production of infectious particles by $97 \%$ and liposome POPG reduced this number by $>99 \%$ (Figure 7D). In addition to shaminfected controls, mice were treated with liposome POPG or $n P O P G$ in the absence of virus (Figure $7 \mathrm{~A}-\mathrm{C}$ ). Mice given only liposome POPG or nPOPG did not show any significantly enhanced inflammatory cell migration into the lungs or increased IFN- $\gamma$ production in the recovered BALF. 
A
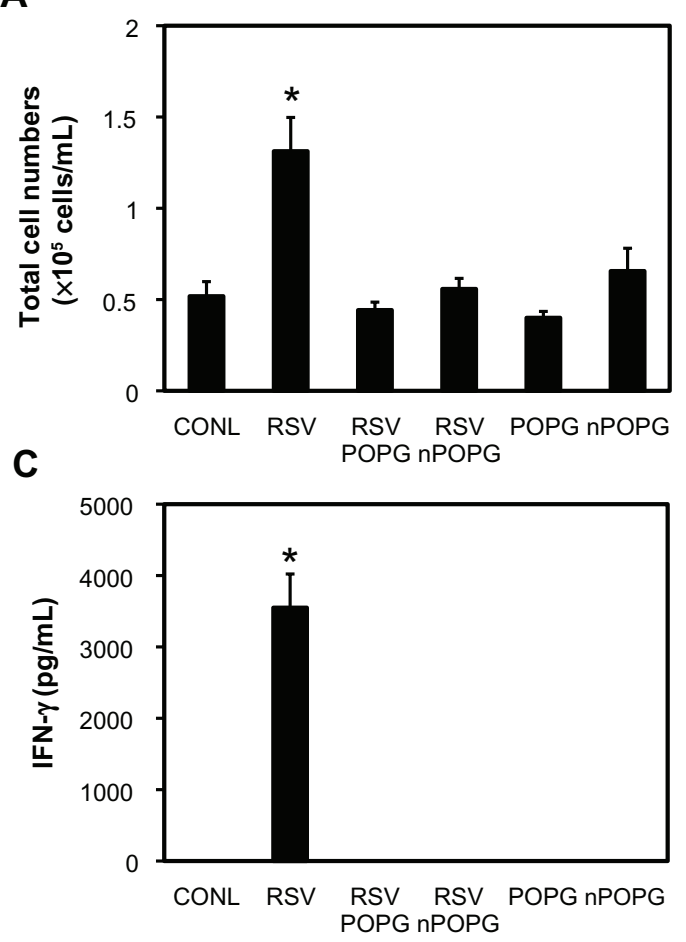

B

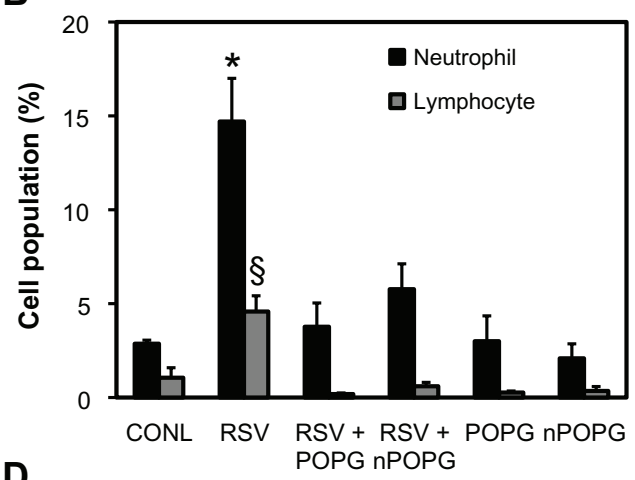

D

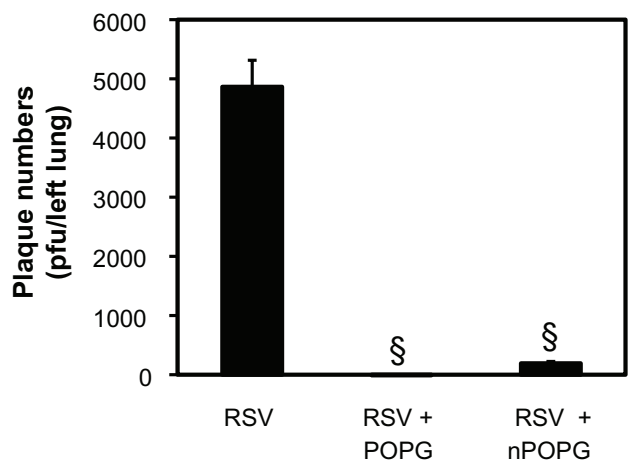

Figure $7 \mathrm{nPOPG}$ inhibits RSV infection and inflammation in vivo. BALB/c mice were infected with RSV $\left(\mathrm{I} \times 10^{7}\right.$ pfu/mouse $)$ by intranasal inoculation in either the absence or presence of liposome POPG or nPOPG ( $400 \mu \mathrm{g}$ phospholipid), as indicated. The mice were euthanized 5 days after infection and their lungs were lavaged and subsequently removed for performing histopathology and quantitative plaque assays. The lavage material was used for analysis of cell populations and quantification of IFN- $\gamma$. (Panel $\mathbf{A}$ ) shows the total cell numbers in BALF. (Panel B) shows the inflammatory cell populations in BALF. (Panel C) shows the IFN- $\gamma$ levels in BALF as determined by ELISA. (Panel D) shows RSV titer in tissue homogenates prepared from the left lung in each group as measured by quantitative plaque assay.

Notes: Values shown are the means \pm SE for two independent experiments: ${ }^{*} P<0.00 \mathrm{I},{ }^{\$} P<5 \times 10^{-5}$. In each experiment, every group contained five to eight mice.

Abbreviations: CONL, uninfected control; nPOPG, nanodisc palmitoyl-oleoyl-phosphatidylglcyerol; POPG, liposomal palmitoyl-oleoyl-phosphatidylglycerol; RSV, respiratory syncytial virus; SE, standard errors of the mean.

We also examined the effects of nPOPG on lung histology, as previously described, for liposome POPG. ${ }^{23,24}$ The lung histology score of control animals was $2.1 \pm 0.3$, which was increased to $8.3 \pm 0.7$ by viral infection. As shown in Figure 8A, nPOPG reduced the virus effect on the histopathology score to $2.5 \pm 0.5$. In addition, treatment with nPOPG alone did not induce any inflammatory response. Figure 8B shows representative micrographs, which reveal the presence of lymphocytes and neutrophils in peribronchial and alveolar compartments in response to RSV infection. In contrast to the results obtained with RSV treatment alone, the inclusion of nPOPG with the virus almost completely suppressed the inflammatory histopathology. The histopathology results obtained with nPOPG are nearly identical to those previously reported for liposome POPG. ${ }^{23}$ In addition, treatment of the mice with nPOPG alone failed to produce evidence of significant inflammation in the tissue sections. Collectively, the above findings demonstrate that $\mathrm{nPOPG}$ is potent as a viral antagonist in mice.

\section{nPOPG turnover in the lung}

\section{is rapid in vivo}

We examined the in vivo turnover of $\mathrm{nPOPG}$ by measuring the amount of the MSP component of the nanodisc in BALF after intranasal inoculation. We inoculated $400 \mu \mathrm{g}$ of $\mathrm{nPOPG}$ into mice and collected BALF at various time points and performed quantitative immunoblotting for MSP using a human Apo A-I antibody, as shown in Figure 9A. The time-dependent recovery of the Apo A-I was normalized to the initial amounts recovered immediately after inoculation. A strong Apo A-I signal was detected immediately following the inoculation and persisted through the 30-minutes and 1-hour time points. At 3 and $6 \mathrm{~h}$ after inoculation, the Apo A-I was either very low or undetectable in the mouse lavage. These results indicate a relatively rapid turnover for the $\mathrm{nPOPG}$ in mice and suggest a therapeutic window for $\mathrm{nPOPG}$ of approximately $1-2$ hours in mice.

\section{Discussion}

In this report we provide evidence of an anti-inflammatory and anti-viral effect of nanodiscs assembled from MSP 
A

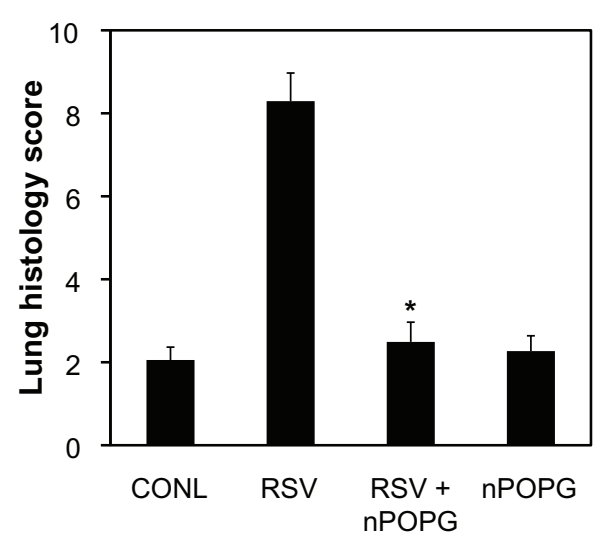

B

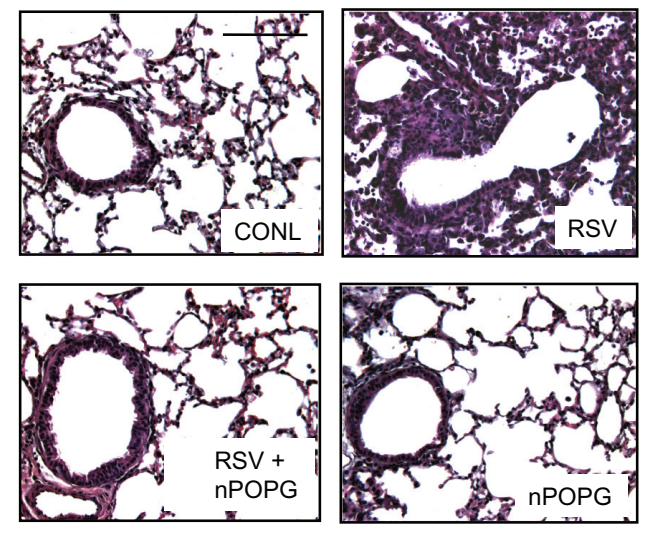

Figure 8 nPOPG suppresses lung inflammation elicited by RSV. (A) Lungs from sham-infected (CONL) or RSV-infected mice treated with nPOPG were fixed in I0\% buffered formalin, embedded in paraffin, and stained with hematoxylineosin. Lung sections from each animal were evaluated and given a histopathology score. Values shown are the means $\pm S E$. $* P=4.1 \times 10^{-6}$. (B) Representative micrographs from the CONL- and virus-infected groups treated with $400 \mu \mathrm{g} / \mathrm{mL} \mathrm{nPOPG}$.

Note: The bar inset in the CONL panels corresponds to $200 \mu \mathrm{m}$.

Abbreviations: CONL, uninfected control; nPOPG, nanodisc palmitoyl-oleoyl-phosphatidylglycerol; RSV, respiratory syncytial virus; SE, standard errors of the mean.

and the phospholipid POPG. In vitro studies revealed that nPOPG suppresses RSV-elicited IL-8 production with an $\mathrm{IC}_{50}$ of $19.3 \mu \mathrm{g} / \mathrm{mL}$; this level is comparable to the value of $12.3 \mu \mathrm{g} / \mathrm{mL}$ obtained with liposomes. The findings demonstrate that $\mathrm{nPOPG}$ is effective at preventing the release of one of the earliest cytokines expressed by epithelial cells in response to viral infection. In vivo, IL-8 acts as a potent neutrophil chemotactic factor. The in vitro inhibition of viral plaque formation by $\mathrm{nPOPG}$ shows that concentrations of $200 \mu \mathrm{g} / \mathrm{mL}$ lipids suppress viral infection by $93 \%$; this potency is nearly equivalent to that obtained with liposome POPG. This suppression of plaque formation is primarily a consequence of nPOPG inhibiting the attachment of the virus to the surface of epithelial cells. The in vitro viral binding experiments show that nPOPG is somewhat more effective than liposome POPG. Consistent with the findings that the viral attachment step is disrupted by $\mathrm{nPOPG}$ are the results demonstrating a reduction in viral F-protein mRNA production by a factor of 100 .

The in vitro findings for $\mathrm{nPOPG}$ are also recapitulated in animal models of RSV infection. RSV challenges to
A

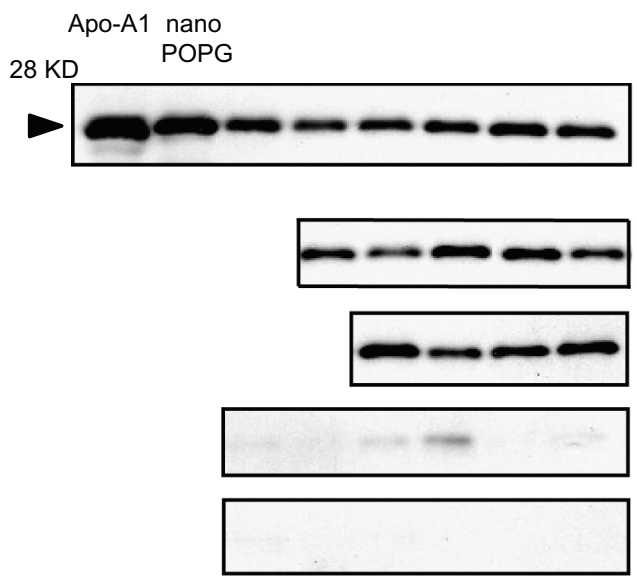

B

(hours)

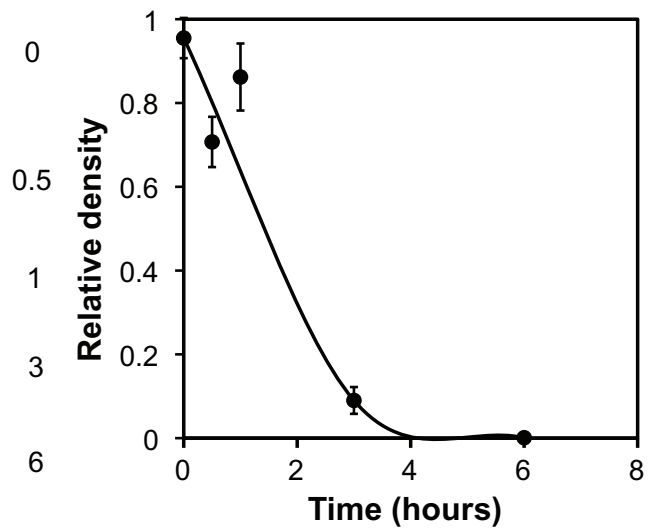

Figure 9 In vivo turnover of nPOPG in the lung. (A) Mice were anesthetized and inoculated with $400 \mu \mathrm{nPOPG}$ and euthanized at the indicated times. The euthanized animals were lavaged with one $\mathrm{mL}$ of PBS and the cell-free recovered material was subjected to electrophoresis and quantitative immunoblotting using anti-human Apo A-I antibodies. Samples from all time points were diluted 20 -fold from the original BALF sample. (B) Images in (A) were quantified using NIH-Image J software.

Note: Values are shown as the means \pm SE for three independent experiments, with four to eight mice/time point in each experiment.

Abbreviations: KD, kilo Dalton; nano POPG, nanodisc palmitoyl-oleoyl-phosphatidylglycerol; RSV, respiratory syncytial virus; SE, standard errors of the mean. 
mice demonstrate that $400 \mu \mathrm{g} / \mathrm{mL}$ nPOPG suppresses IFN- $\gamma$ production and reduces the viral burden in the lung by $96 \%$; similar effects are obtained with liposome POPG. The reduction of the in vivo IFN- $\gamma$ response on day 5 , near the peak of viral infection, clearly indicates that the viral infection has largely been unsuccessful. Consistent with this conclusion are the histopathological results demonstrating that nPOPG potently suppresses RSV-induced lung inflammation. The lack of significant increases in neutrophils in the lungs of mice treated with RSV and $n P O P G$ indicates in vivo suppression of the production of keratinocyte chemoattractant, the mouse counterpart of IL-8. Collectively, these findings demonstrate that nPOPG has the potential to be a useful new type of drug delivery platform and an anti-viral agent against RSV, capable of significantly limiting infection and inflammation.

The Apo A-I content of nPOPG provides a convenient molecular marker for following the turnover of nanodiscs introduced into the lung by intranasal inoculation. Our results demonstrate that the apparent biological half-life for the protein component of the nanodiscs is between 60-120 min. This turnover rate indicates that $n P O P G$ will only have a narrow window of efficacy in the mouse. However, the turnover of pulmonary surfactant proteins and lipids in the extracellular alveolar compartment is generally thought to be dependent upon the respiratory rate. ${ }^{29}$ In mice this respiratory rate is $250-300$ breaths $/ \mathrm{min}$, whereas in humans this value is 18 breaths/minute in adults and 25-30 breaths/min in newborns. These differences in respiratory rate suggest that the turnover time of $n P O P G$ may increase by as much as 10-fold in humans compared to mice. A turnover halftime of $\sim 600$ minutes in humans is a practical therapeutic window. The extracellular nPOPG action site requires that the extracellular pools of the lipid be maintained at adequate levels to prevent newly emergent viruses from spreading to the uninfected cells. Our experimental design utilized a single inoculation of nanodiscs in mice, as we wanted to avoid multiple rounds of anesthesia. Potential translation of the nPOPG system to humans might easily accommodate multiple dosing regimens within a 24-hour period. If higher levels of nPOPG can be maintained over longer time periods in humans, then such treatments should be effective for both prophylaxis and post-infection treatment.

Overall, our data show that nPOPG is comparable to liposome POPG in its anti-inflammatory and anti-viral properties. However, the $\mathrm{nPOPG}$ particles are $\sim 10 \mathrm{~nm} \times 5 \mathrm{~nm}$ in dimension and contain approximately 130 phospholipid molecules/particle, whereas the POPG liposomes are hollow spheres $25-50 \mathrm{~nm}$ in diameter that contain between $5 \times 10^{3}$ and $2 \times 10^{4}$ phospholipid molecules per particle. The smaller nanodisc size may offer some significant advantages for delivery to the lungs by inhalation. The MSP scaffold for nPOPG also provides a protein platform for modification that could potentially be manipulated to enhance the biological half-life or the targeting of the particles.

In summary, our data show that nanodiscs selfassembled with the therapeutic phospholipid POPG (nPOPG) effectively inhibit RSV infection and the resulting inflammatory response, both in vitro and in vivo. These findings suggest that nanodiscs may be a useful platform for the delivery of a range of bioactive lipids for therapeutic purposes.

\section{Acknowledgment}

This work was supported by NIH grant HL094629 and Colorado Bioscience Discovery Grant-2009 to DRV, and by NIH grants GM33775 and GM31756 to SGS.

\section{Disclosure}

The authors report no conflicts of interest.

\section{References}

1. Bayburt TH, Grinkova YV, Sligar SG. Self-assembly of discoidal phospholipid bilayer nanoparticles with membrane scaffold proteins. Nano Letters. 2002;2(8):853-856.

2. Denisov IG, Grinkova YV, Lazarides AA, Sligar SG. Directed selfassembly of monodisperse phospholipid bilayer nanodiscs with controlled size. J Am Chem Soc. 2004;126(11):3477-3487.

3. Grinkova YV, Denisov IG, Sligar SG. Engineering extended membrane scaffold proteins for self-assembly of soluble nanoscale lipid bilayers Protein Engineering, Design and Selection. 2010;23(11):843-848. doi: 10.1093/protein/gzq060.

4. Atkinson D, Small DM. Recombinant lipoproteins: implications for structure and assembly of native lipoproteins. Annu Rev Biophys Biophys Chem. 1986;15:403-456.

5. Atkinson D, Smith HM, Dickson J, Austin JP. Interaction of apoprotein from porcine high-density lipoprotein with dimyristoyl lecithin. 1. The structure of the complexes. Eur J Biochem. 1976;64(2): 541-547.

6. Brouillette CG, Anantharamaiah GM, Engler JA, Borhani DW. Structural models of human apolipoprotein A-I: a critical analysis and review. Biochim Biophys Acta. 2001;1531(1-2):4-46.

7. Brouillette CG, Jones JL, Ng TC, Kercret H, Chung BH, Segrest JP. Structural studies of apolipoprotein A-I/phosphatidylcholine recombinants by high-field proton NMR, nondenaturing gradient gel electrophoresis, and electron microscopy. Biochemistry. 1984;23(2): 359-367.

8. Jonas A. Reconstitution of high-density lipoproteins. Methods Enzymol. 1986;128:553-582.

9. Matz CE, Jonas A. Micellar complexes of human apolipoprotein A-I with phosphatidylcholines and cholesterol prepared from cholate-lipid dispersions. J Biol Chem. 1982;257(8):4535-4540.

10. Segrest JP. Amphipathic helixes and plasma lipoproteins: thermodynamic and geometric considerations. Chem Phys Lipids. 1977;18(1): $7-22$. 
11. Wlodawer A, Segrest JP, Chung BH, Chiovetti R Jr, Weinstein JN. High-density lipoprotein recombinants: evidence for a bicycle tire micelle structure obtained by neutron scattering and electron microscopy. FEBS Lett. 1979;104(2):231-235.

12. Fielding CJ, Fielding PE. Dynamics of lipoprotein transport in the circulatory system. In: Vance DE, Vance JE, editors. Biochemistry of Lipids, Lipoproteins and Membranes, 5th ed. Amsterdam: Elsevier; 2008:533-553.

13. Zannis VI, Chroni A, Krieger M. Role of apoA-I, ABCA1, LCAT, and SR-BI in the biogenesis of HDL. J Mol Med (Berl). 2006; 84(4):276-294.

14. Marcel YL, Kiss RS. Structure-function relationships of apolipoprotein A-I: a flexible protein with dynamic lipid associations. Curr Opin Lipidol. 2003;14(2):151-157.

15. Nath A, Atkins WM, Sligar SG. Applications of phospholipid bilayer nanodiscs in the study of membranes and membrane proteins. Biochemistry. 2007;46(8):2059-2069.

16. Sligar SG. Finding a single-molecule solution for membrane proteins. Biochem Biophys Res Commun. 2003;312(1):115-119.

17. Ritchie TK, Grinkova YV, Bayburt TH, et al. Reconstitution of membrane proteins in phospholipid bilayer nanodiscs. Methods Enzymol. 2009;464:211-231.

18. Bayburt TH, Sligar SG. Membrane protein assembly into nanodisks. FEBS Letters. 2010;584(9):1721-1727.

19. Denisov IG, Sligar SG. Cytochromes P 450 in nanodiscs. Biochim Biophys Acta. 2011;1814(1):223-229.

20. Simoes EA. Respiratory syncytial virus infection. Lancet. 1999; 354(9181):847-852.

21. Falsey AR, Hennessey PA, Formica MA, Cox C, Walsh EE. Respiratory syncytial virus infection in elderly and high-risk adults. $N$ Engl J Med. 2005;352(17):1749-1759.
22. Hall CB. Respiratory syncytial virus and parainfluenza virus. $N$ Engl J Med. 2001;344(25):1917-1928.

23. Numata M, Chu HW, Dakhama A, Voelker DR. Pulmonary surfactant phosphatidylglycerol inhibits respiratory syncytial virus-induced inflammation and infection. Proc Natl Acad Sci U S A. 2010; 107(1):320-325

24. Cimolai N, Taylor GP, Mah D, Morrison BJ. Definition and application of a histopathological scoring scheme for an animal model of acute Mycoplasma pneumoniae pulmonary infection. Microbiol Immunol. 1992;36(5):465-478.

25. Dakhama A, Park JW, Taube C, et al. The enhancement or prevention of airway hyperresponsiveness during reinfection with respiratory syncytial virus is critically dependent on the age at first infection and IL-13 production. J Immunol. 2005;175(3):1876-1883.

26. LeVine AM, Elliott J, Whitsett JA, et al. Surfactant protein-D enhances phagocytosis and pulmonary clearance of respiratory syncytial virus. Am J Respir Cell Mol Biol. 2004;31(2):193-199.

27. McKimm-Breschkin JL. A simplified plaque assay for respiratory syncytial virus - direct visualization of plaques without immunostaining. J Virol Methods. 2004;120(1):113-117.

28. Numata MKP, Nagashima Y, Posey J, Hartshorn K, Woodland D, Voelker DR. Phosphatidylglycerol suppresses influenza A virus infection. Am J Respir Cell Mol Biol. 2012;46:479-487.

29. Hallman M, Merritt TA, Bry K. The fate of exogenous surfactant in neonates with respiratory distress syndrome. Clin Pharmacokinet. 1994;26(3):215-232.

30. Shih, AY, Denisov IG, Phillips JC, Sligar SG, Schulten K. Molecular dynamics simulations of discoidal bilayers assembled from truncated human lipoproteins. Biophys J. 2005;88:548-556.
International Journal of Nanomedicine

\section{Publish your work in this journal}

The International Journal of Nanomedicine is an international, peerreviewed journal focusing on the application of nanotechnology in diagnostics, therapeutics, and drug delivery systems throughou the biomedical field. This journal is indexed on PubMed Central, MedLine, CAS, SciSearch $₫$, Current Contents $₫ /$ Clinical Medicine,

\section{Dovepress}

Journal Citation Reports/Science Edition, EMBase, Scopus and the Elsevier Bibliographic databases. The manuscript management system is completely online and includes a very quick and fair peer-review system, which is all easy to use. Visit http://www.dovepress.com/ testimonials.php to read real quotes from published authors. 